\title{
Association between 24-hour movement behaviors and health-related quality of life in children
}

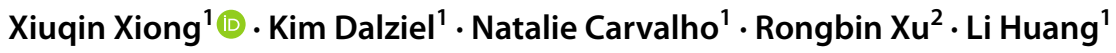

Accepted: 28 May 2021 / Published online: 3 June 2021

(c) The Author(s), under exclusive licence to Springer Nature Switzerland AG 2021

\begin{abstract}
Purpose To assess the associations between adherence to 24-hour movement behaviors guidelines and child general health and functional status measured by health-related quality of life.

Methods The Longitudinal Study of Australian Children (2004-2016) a nationally representative sample with data available for children aged 2-15 years was used. Physical activity time, recreational screen time, and sleep time were calculated from time use diaries and classified as 'meeting guidelines' or 'not' based on the age-specific 24-h movement guidelines. Child general health and functional status were measured using the multidimensional Pediatric Quality of Life Inventory (PedsQL). Associations between meeting guidelines and PedsQL were assessed using linear mixed effects models.

Results 8919 children were included. Each additional guideline met was associated with a $0.52(95 \%$ confidence interval [CI] 0.39-0.65) increase in PedsQL total score. Compared with meeting no guidelines, the effect of meeting physical activity guidelines alone ( $\beta=0.93,95 \%$ CI $0.42-1.44)$ was larger compared to meeting screen ( $\beta=0.66,95 \%$ CI $0.06-1.27)$ or sleep time ( $\beta=0.47,95 \%$ CI 0.04-0.89) guidelines alone. The highest increment was observed in meeting both screen time and physical activity guidelines $(\beta=1.89,95 \%$ CI 1.36-2.43). Associations were stronger in children from lower-income families ( $\beta$ for meeting all versus none $=2.88,95 \%$ CI 1.77-3.99) and children aged $14-15$ years $(\beta=4.44,95 \%$ CI 2.49-6.40). Conclusions The integration of screen time and physical activity guidelines is associated with the highest PedsQL improvement. The association between guidelines adherence and PedsQL appears stronger for adolescents and those from low-income families.
\end{abstract}

Keywords Physical activity $\cdot$ Screen use $\cdot$ Sleep $\cdot 24$-Hour movement guidelines $\cdot$ Health status $\cdot$ Child $\cdot$ Adolescents . Australia, health equity

\section{Plain English Summary}

The latest National Child Health Poll in Australia showed that the number one health concern for parents in 2021 is 'excessive screen time'. And 'not enough exercise' was also one of the top ten concerns. The existing literature has focused on the single time use behavior and the associated health outcomes. A paradigm shift from individual activity

Xiuqin Xiong

xiuqinx@student.unimelb.edu.au

1 Centre for Health Policy, Melbourne School of Population and Global Health, The University of Melbourne, Parkville, VIC, Australia

2 Department of Epidemiology and Preventive Medicine, School of Public Health and Preventive Medicine, Monash University, Melbourne, VIC, Australia components to the 24-hour time use is critical. We explored the relationship between meeting the integrated 24-hour movement guidelines including recreational screen use, physical activity and sleep, and health-related quality of life (HRQOL) by age group and socio-economic status. We found a positive association between adherence to 24-hour movement guidelines and HRQOL using a nationally represented sample of nearly 9,000 children aged 2-15 years in Australia. Meeting physical activity guidelines alone contributed more to HRQOL than meeting sleep and recreational screen time guidelines alone respectively. The HRQOL improvement was the highest for children aged 14-15 years and those from lower income families. This has important implications for policy makers seeking to promote child health with modifiable behaviors. The impact of the guidelines varies for different ages which highlighted the 
necessities of having guidelines for specific development stages.

During 2010 to 2012, national recommendations for physical activity and sedentary behavior in children were released in Australia, the UK, and Canada [1-4]. The recommendations in the movement behaviors are backed by evidence from physical inactivity [5-7], excessive digital media use [8,9], insufficient sleep [10, 11], and their associated unfavorably physical, psychological, social, and cognitive health outcomes. In 2016, Canada was the first country to release integrated 24-h movement guidelines (a mix of physical activity, screen use and sleep in each 24-h period, please see Online Appendix 1 for details) for school-aged children and youth [12], followed by the Australian Government in 2017, New Zealand, South Africa, the UK, the USA, and by the World Health Organization (WHO) [13]. The 24-h movement paradigm shifts the focus from individual activity components to the whole period and emphasizes the interactions between activities during a day. For example, time spent on physical activity has its own health benefit, but it also has positive impacts on sleep [14] and screen time [15], while sleep and screen time interact with each other too [16]. The guidelines from all jurisdictions are broadly equivalent regarding the recommended time per activity, with only minor variations in wording and use of 4 or 5 years as the age cutoff for early years [13]. Taking physical activity as an example, the WHO recommends that children and adolescents aged 5-17 years should do at least an average of $60 \mathrm{~min}$ of moderate to vigorous physical activity per day across the week, mostly aerobic (see Online Appendix 1 for the detailed age-specific guidelines). The 24-h guideline was evidence-based and supported by systematic reviews examining the relationships between and among movement behaviors, stakeholder survey, and focus groups/stakeholder interviews.

Nevertheless, evidence on the association between adherence to 24-h guideline and child general health outcomes in a population representative sample is lacking. Traditionally, health impact was primarily measured using mortality and morbidity. At the present time, the importance of healthrelated quality of life (HRQOL) beyond survival is widely recognized by clinicians, researchers, and policy makers to assist care management and policy decisions. [17-19] Advantages of using HRQOL include evaluating the overall influence of health conditions on individual's life and capturing clinical and non-clinical benefits of care such as symptom relief $[20,21]$. The multidimensional construct of HRQOL often includes physical, emotional, and social dimensions of health [17], assessing the self-reported health status and impact of health as opposed to objective biological indicators and diagnoses. HRQOL instruments can be generic or condition specific [19], with some classified as non-preference-based (e.g., PedsQL, KIDSCREEN), while others preference-based (e.g., EQ-5D-Y, CHU9D). Nonpreference-based instruments are mainly applied in clinical studies, population health studies, etc., while preferencebased measurements are mainly used in economic evaluations [20].

Children's HRQOL could be affected by many factors, such as socioeconomic status [22], special health care needs [23], or physical activity [5]. There are a number of contributors that are also related to children's physical activity, such as socioeconomic status [24], parental physical activity [25], and family environment [26]. Several studies have investigated the relationship between HRQOL and physical activity, screen use, and sleep separately $[5,9,27]$ or a combination of two of them in selected age groups of children $[28,29]$. To the best of our knowledge, only two previous studies directly investigated the association between 24-h movement behaviors and HRQOL [30, 31]. In a multinational, observational study of children aged 9-11 years, children had significantly higher HRQOL when they met the screen time recommendation only, the screen plus sleep recommendations, and all three recommendations compared with those who met none [30]. Another study followed children aged 3-5 until they were aged 9-11 years and found no significant associations between baseline 24-h movement behaviors (capturing physical activity, screen use and sleep) and later HRQOL among 471 children [31]. The two studies had either a narrow age range or a relatively small sample with the majority of the participants from urban or relatively high socioeconomic background families. Also, children's recreational screen time or sleep time in these studies was obtained from survey questionnaires rather than a time use diary, which may be subject to recall or social desirability bias [32, 33].

This study aims to investigate how adherence to the integrated 24-h movement guidelines was associated with HRQOL in a population representative sample with children aged $2-15$ years. We used a nationally representative sample with a comprehensive age range and time use diaries which allowed comparison between different age groups and more accurate estimates to complement the existing evidence.

\section{Methods}

\section{Study design and participants}

Data from all seven waves of the Longitudinal Study of Australian Children (LSAC) were used. The LSAC data was a de-identified, publicly available, existing dataset provided by 
the Department of Social Services, the Australian Institute of Family Studies, and the Australian Bureau of Statistics. The LSAC, which commenced in 2004, involves repeated biennial assessment ('waves') of over 10,000 children across two age cohorts (a birth cohort of 5,107 children aged 0-1 year in 2003-2004, and a kindergarten cohort of 4,983 children aged 4-5 years in 2003-2004). The LSAC used a two-stage cluster randomized design with stratification by state and then by major metropolitan center to obtain a geographically representative sample of children and their families. The LSAC sampling design and field methods are detailed elsewhere [34]. Each wave was approved by The Australian Institute of Family Studies Ethics Committee and families provided written informed consent.

\section{Time use data}

Physical activity, recreational screen use, and sleep time were derived from the time use diaries which documented 24-h use of time for each child. We have focused on children aged 2-15 years to align to the period when HRQOL and time use data were available.

Two types of time use diaries were available: a parentcompleted diary for children aged 2-9 years and a childcompleted diary for those aged 10-15 years. For the parentcompleted diaries, the primary caregiver, usually the mother, completed two diaries with one for a weekday and one for a weekend day. The primary carer was asked to complete a diary the weekday immediately following an interview, as well as one weekend diary randomly selected by the interviewer to achieve a random allocation of weekdays and a random allocation of weekend days [35]. They were asked to record what the child was doing in 15-min blocks of time, from a list of pre-coded activities in 96 blocks throughout the day [35]. The average daily recreational screen use, physical activity, and sleep time were calculated as a weighted average of the week. The average missingness of the 96 time blocks was $6.56 \%$ for children aged 2-9 years. Individual's missing entries in the 96 blocks were imputed by age, sex, socioeconomic status, day of the week, wave, cohort, and time block of the day using random forest technique [36]. Based on the complete dataset without time use missing values, the accuracy of the imputation models compared with the observed time use ranged from 82 to $99 \%$.

Children aged 10-15 years old were required to selfrecord their own time use the day before the scheduled interview. The diary included the start time of each activity, and sleep and awake time [37]. The time use was electronically recorded by an interviewer the next day during the interview so that any uncertain entries could be clarified. Due to the concerns of survey burden, each child was required to complete only one diary on a randomly selected day by the interviewer [37]. Missingness of the time use data for sleep, screen, and physical activities ranged from 0.06 to $1.52 \%$. This was considered to be minimal and we did not impute the missing data for children aged $10-15$ years.

Based on the time use data, we defined adherence to guidelines on physical activity, recreational screen use, and sleep according to the age-specific 24-h movement guidelines (see Online Appendix 1 for details). The time use diaries were not collected from the children aged 6-9 years (wave 4 and 5) of the birth cohort, where data from the kindergarten cohort (6-9 years from wave 2 and 3 ) alone was used.

\section{Health-related quality of life}

We used the Pediatric Quality of Life Inventory (PedsQL) Version 4.0, an established, standardized, and generic instrument [17] for HRQOL assessment in children and adolescents available across the age ranges of 2-18 years. The PedsQL measures four health dimensions: physical, emotional, social, and school functioning [17]. There are 23 items (21 items for 2-4 years) in the PedsQL Inventory. In the LSAC, PedsQL was filled out by the study child's primary caregiver, who rated the frequency of each item in the past month with a 5-point Likert scale from 0 (Never) to 4 (Almost always). Items are reversed scored and linearly transformed to a $0-100$ scale $(0=100,1=75,2=50,3=25$, $4=0$ ) in the PedsQL, with higher scores indicating better HRQOL [17]. The total score was calculated as the sum of the score of each item divided by the number of items answered. If more than $50 \%$ of the items are missing, the total score should not be computed. A 4.5 points change in the total score for parent proxy report is considered to be clinically meaningful [17].

\section{Statistical analysis}

Covariates considered to be associated with HRQOL were controlled for, including age, sex, indigenous status, language spoken at home, number of siblings, household income, and parental education [38-40]. Age was grouped into $2-4,5-13$, and $14-15$ years corresponding to the $24-\mathrm{h}$ guideline categories. Language spoken at home was English or otherwise. Number of siblings was categorized as single child, one sibling, two or more. Household income was categorized as lowest $25 \%$, middle $50 \%$, and highest $25 \%$ using quartiles in each wave cohort. Highest parental education was whether any parent has bachelor's degree or above. We also adjusted for children's general health using a 2-question sequence Children With Special Health Care Needs screener [41]. The screener identified children having more than average health care needs that is expected to last 
more than 12 months, implicating an estimated $16 \%$ of the Australian children under 18 years of age [41].

Linear mixed modeling (or multi-level model) with random intercept for individuals was used to evaluate the association between meeting 24-h movement guidelines and HRQOL [42]. The random intercept accounts for repeated measurements of individuals by defining the child identifier as the cluster variable [43]. The coefficients of the linear mixed effects model can be interpreted as the average difference in HRQOL in response to both within- and the interindividual changes [44-46].

Two regressions were run in the primary analysis: one treating guideline adherence as a continuous variable (number of guidelines met: $0,1,2,3$ ) and the other as a categorical variable (meeting different combinations of three guidelines: none, sleep, screen, physical activity, sleep and screen, sleep and physical activity, screen and physical activity, all three). To explore potential heterogeneity, subgroup analyses were conducted by age, sex, and household income for all children included and by whether it was a school day (school day for children aged 4-15 years). All covariates in the primary analysis except for the grouping variable were included in the subgroup analyses. We also performed a sensitivity analysis by repeating our main analysis using the data without imputation of the missing values.

The Stata statistical software package (version 16.0, College Station, Texas) was used for data cleaning and analysis, and R (version 3.5.3, "caret" package) was used for data imputation.

\section{Results}

Observations with the outcome variable PedsQL missing were dropped, leaving 33,168 person-wave observations from 8,874 children aged $2-15$ years old. The demographic characteristics, average time per activity, 24-h guideline adherence, and average PedsQL scores are described by age group in Table 1. Overall, physical activity and sleep time were lower in older children, and recreational screen time was higher in older children. Of the three guidelines, an average of 1.6 (SD 0.9) guidelines were met by children aged 2-15 years, $16.6 \%$ of children met all three guidelines, and $10.2 \%$ met none. The mean of the PedsQL total score was slightly lower in older age groups.

Table 1 Key characteristics of the sample at person-year response level

\begin{tabular}{|c|c|c|c|c|}
\hline & $2-4$ years $(N=6749)$ & $5-13$ years $(N=8246)$ & $\begin{array}{l}14-15 \text { years } \\
(N=3075)\end{array}$ & $\begin{array}{l}\text { Total } \\
(N=8919)\end{array}$ \\
\hline \multicolumn{5}{|l|}{ Demographics } \\
\hline Special health care needs, yes & 12.8 & 15.8 & 19.6 & 15.4 \\
\hline Female, yes & 48.4 & 49.0 & 49.7 & 48.9 \\
\hline Indigenous, yes & 2.3 & 2.4 & 2.0 & 2.4 \\
\hline Speaking English at home, yes & 91.5 & 90.7 & 90.2 & 90.9 \\
\hline Two parent family, yes & 90.7 & 86.2 & 83.4 & 87.1 \\
\hline Parental education bachelor or above, yes & 46.8 & 46.4 & 46.8 & 46.6 \\
\hline \multicolumn{5}{|l|}{ Number of siblings } \\
\hline Single child & 14.0 & 8.8 & 11.6 & 10.4 \\
\hline 1 sibling & 51.4 & 45.7 & 46.7 & 47.3 \\
\hline$\geq 2$ siblings & 34.7 & 45.5 & 41.6 & 42.3 \\
\hline Household income (AU\$1,000 per week) & $1.3(0.9)$ & $2.0(1.5)$ & $2.5(1.7)$ & $1.9(1.5)$ \\
\hline \multicolumn{5}{|l|}{ Outcomes } \\
\hline \multicolumn{5}{|l|}{ Time use } \\
\hline Screen time (hours per day) & $2.0(1.4)$ & $2.7(2.2)$ & $3.7(3.0)$ & - \\
\hline Physical activity (hours per day) & $2.1(1.5)$ & $1.8(1.6)$ & $1.1(1.4)$ & - \\
\hline Sleep time (hours per day) & $11.3(1.9)$ & $10.0(1.4)$ & $9.1(1.6)$ & - \\
\hline \multicolumn{5}{|l|}{ 24-h guidelines adherence } \\
\hline Number of guidelines met & $1.3(0.7)$ & $1.8(0.9)$ & $1.3(0.9)$ & $1.6(0.9)$ \\
\hline Percentage meeting three guidelines & 5.4 & 22.1 & 10.7 & 16.6 \\
\hline Percentage meeting no guideline & 11.2 & 8.5 & 19.1 & 10.2 \\
\hline PedsQL total score & $81.9(10.1)$ & 79.3(13.1) & 78.5(14.6) & $79.9(12.6)$ \\
\hline
\end{tabular}

Data are $\%$ or mean (SD). The percentages are calculated based on person-wave observations. $N$ is the number of unique children. Screen time was recreational screen time. According to $24-\mathrm{h}$ movement guidelines, total sleep time was used for 0-4 years, and sleep time at night was used for 5-15 years. Household income prior to 2016 was inflated to 2016 Australian dollars. PedsQL total score ranges from 0 to 100 points 


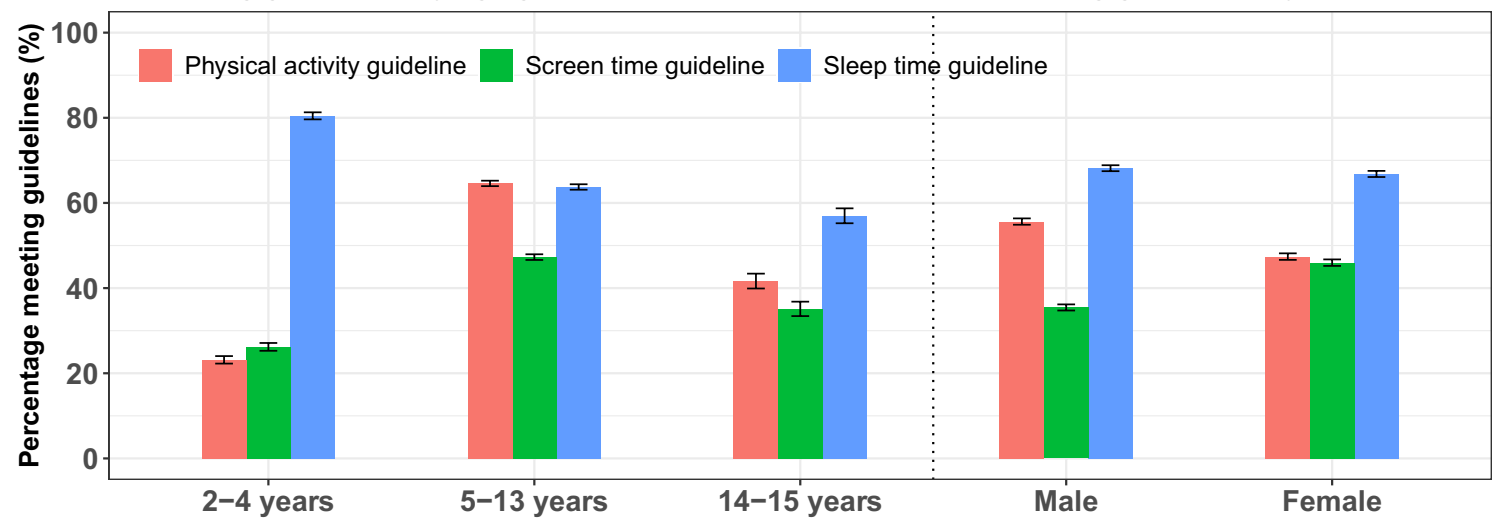

C. Meeting guidelines by household income

D. Meeting guidelines by day type

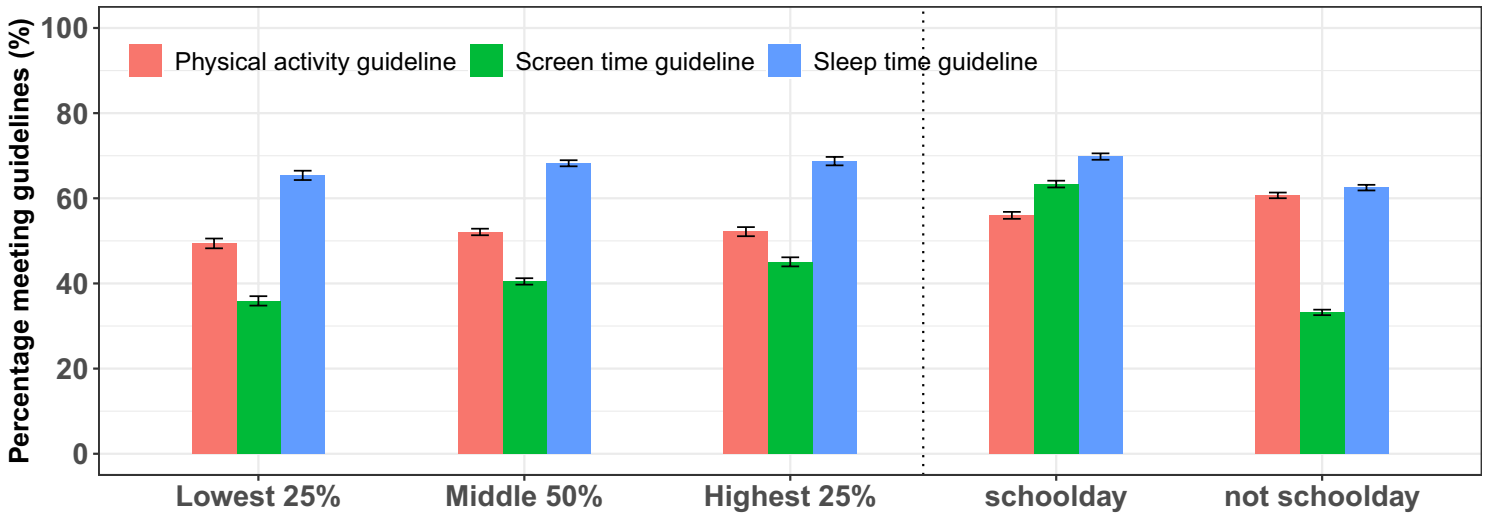

Fig. 1 Single movement guideline adherence. Note 'Whether a school-day' data are available for 4-15 years old. All other data are available for 2-15 years old. The error bars represent the $95 \%$ confidence intervals of the percentages of guidelines adherence

Single guideline adherence for all children and by subgroups is presented in Fig. 1. Overall, adherence to recreational screen time recommendation was the lowest. Boys had relatively low adherence to screen time guidelines, while girls had lower adherence to physical activity guidelines. Children from the lowest household income families had the lowest percentages of adherence to all three guidelines. On non-schooldays, screen time guideline adherence was much lower compared to schooldays.

The regression analysis showed that HRQOL was positively associated with the number of guidelines met, and the PedsQL total score was 0.52 (95\%CI 0.39-0.65) higher with each additional guideline met (Table 2). When 'guideline adherence' was treated as a categorical variable, the PedsQL total score was 1.61(95\%CI 1.16-2.07) higher for children meeting all three guidelines compared with those meeting none. Meeting physical activity guidelines alone had similar effect with meeting both screen and sleep guidelines $(\beta=0.93,95 \% \mathrm{CI} 0.42-1.44$ versus $\beta=0.83,95 \% \mathrm{CI}$ $0.34-1.32$ ). Meeting screen time and physical activity guidelines was associated with greater QOL improvements ( $\beta=1.89,95 \%$ CI 1.36-2.43) compared with meeting screen time and sleep guidelines $(\beta=0.83,95 \%$ CI $0.34-1.32)$. Results using data without imputation of the missing values were consistent with the main results (Online Appendix 2).

In Fig. 2, guideline adherence was estimated using the total number of guidelines met, while in Fig. 3, adherence was whether each individual guideline was met. The scales of the associations were different by subgroups (Figs. 2, 3). Meeting guidelines was associated with greatest HRQOL improvements for the 14-15 years old ( $\beta$ for meeting all guidelines versus meeting none $=4.44,95 \%$ CI $2.49-6.40$ ) and the lowest $25 \%$ income group $(\beta=2.88,95 \% \mathrm{CI}$ 1.77-3.99). The associated improvements with guideline adherence were lowest in the 2-4 years old and the highest income group. We did not find notable difference by gender and by school day. When meeting all three guidelines compared with meeting none of the guidelines, the HRQOL difference in 14-15 years old children (4.44 points) is approaching the minimal clinically important difference, 4.50 points.

For different combinations of guidelines, the patterns of the associations also differ based on age groups. Figure 3 shows that for 2-4 years old, only meeting all three 
Table 2 Association between meeting 24-h movement guidelines and HRQOL

\begin{tabular}{|c|c|c|c|c|}
\hline & \multicolumn{2}{|c|}{$\begin{array}{l}\text { Adherence to guidelines as continuous vari- } \\
\text { able (model 1) }\end{array}$} & \multicolumn{2}{|c|}{$\begin{array}{l}\text { Adherence to guidelines as categorical } \\
\text { variable (model } 2 \text { ) }\end{array}$} \\
\hline & $\beta$ coefficient $(95 \% \mathrm{CI})$ & $p$ value & $\beta$ coefficient $(95 \% \mathrm{CI})$ & $p$ value \\
\hline Special health care needs (reference: no) & $-5.34(-5.70,-4.97)$ & $<0.001$ & $-5.33(-5.69,-4.97)$ & $<0.001$ \\
\hline Female (reference: male) & $0.28(-0.13,0.69)$ & 0.182 & $0.30(-0.11,0.71)$ & 0.155 \\
\hline Indigenous (reference: no) & $-2.81(-4.07,-1.56)$ & $<0.001$ & $-2.82(-4.08,-1.57)$ & $<0.001$ \\
\hline Speak English at home (reference: no) & $3.01(2.33,3.70)$ & $<0.001$ & $3.00(2.32,3.68)$ & $<0.001$ \\
\hline Two parent family (reference: no) & $2.02(1.53,2.50)$ & $<0.001$ & $2.01(1.52,2.49)$ & $<0.001$ \\
\hline Parental education bachelor or above (reference: no) & $0.34(-0.05,0.72)$ & 0.086 & $0.34(-0.04,0.73)$ & 0.081 \\
\hline \multicolumn{5}{|l|}{ Number of siblings (reference: single child) } \\
\hline One sibling & $0.38(-0.15,0.92)$ & 0.160 & $0.38(-0.16,0.91)$ & 0.166 \\
\hline$\geq 2$ siblings & $1.01(0.44,1.57)$ & $<0.001$ & $1.00(0.44,1.57)$ & 0.001 \\
\hline \multicolumn{5}{|l|}{ Age group (reference: $2-4$ years) } \\
\hline $5-13$ years & $-2.18(-2.44,-1.93)$ & $<0.001$ & $-2.35(-2.62,-2.07)$ & $<0.001$ \\
\hline $14-15$ years & $-2.40(-2.81,-1.98)$ & $<0.001$ & $-2.49(-2.92,-2.07)$ & $<0.001$ \\
\hline \multicolumn{5}{|l|}{ Income group (reference: lowest $25 \%$ ) } \\
\hline Middle $50 \%$ & $0.72(0.36,1.07)$ & $<0.001$ & $0.71(0.36,1.07)$ & $<0.001$ \\
\hline Highest $25 \%$ & $1.27(0.82,1.72)$ & $<0.001$ & $1.26(0.81,1.71)$ & $<0.001$ \\
\hline Number of guidelines met & $0.52(0.39,0.65)$ & $<0.001$ & - & - \\
\hline Guidelines met (reference: none) & - & - & & \\
\hline Sleep only & - & - & $0.47(0.04,0.89)$ & 0.032 \\
\hline Screen time only & - & - & $0.66(0.06,1.27)$ & 0.031 \\
\hline Physical activity only & - & - & $0.93(0.42,1.44)$ & $<0.001$ \\
\hline Screen time + sleep & - & - & $0.83(0.34,1.32)$ & 0.001 \\
\hline Physical activity + sleep & - & - & $1.15(0.71,1.59)$ & $<0.001$ \\
\hline Screen time + physical activity & - & - & $1.89(1.36,2.43)$ & $<0.001$ \\
\hline All met & - & - & $1.61(1.16,2.07)$ & $<0.001$ \\
\hline
\end{tabular}

HRQOL means health-related quality of life. Linear mixed effects model is used. Model 1 treated guidelines adherence as a continuous variable $(x=0,1,2,3)$. Model 2 treated guidelines adherence as a categorical variable $(x=$ none, sleep, screen, physical, sleep + screen, sleep + physical, screen + physical, all three)

guidelines were associated with HRQOL improvement (meeting one or two the guidelines are not associated with an improved HRQOL compared to meeting none). This is in contrast with 5-12 years old where meeting any of the guidelines was associated with improvement. For 14-15 years old, meeting sleep time or recreational screen time guideline alone was not associated with HRQOL improvement.

\section{Discussion}

Understanding the importance of time use for child health is now more important than ever. In 2021, the latest National Child Health Poll in Australia revealed that the number one health concern for parents is excessive screen time with more than $90 \%$ of parents reporting it as a big problem or somewhat of a problem in the community. 'Not enough exercise' was also ranked as the 7th in the top ten health concerns for children. [47] In this study, we found that meeting the recreational screen time plus physical activity guidelines has the strongest positive association with child HRQOL. Meeting the physical activity guidelines alone appears to be more important than meeting other guidelines alone overall and has a greater positive association with HRQOL when in combination with meeting other guidelines. This is consistent with previous systematic reviews which also found that physical activity was most consistently associated with positive health indicators in children aged 5-17 years [48]. One previous study including 3,040 students aged 11-18 years also found that those who were physically active every school day and low screenbased media users had higher HRQOL (PedsQL total score: boys, by 6.6 points; girls, by 7.8 points) compared with the physical inactive and high screen-based media users [28]. One 


\begin{tabular}{|c|c|c|}
\hline Subgroups & & $\beta(95 \% \mathrm{CI})$ \\
\hline Sex & & \\
\hline Male & $\mapsto$ & $0.41(0.22,0.60)$ \\
\hline Female & $\mapsto \bullet$ & $0.63(0.45,0.82)$ \\
\hline Age & & \\
\hline $2-4$ years & $\longmapsto$ & $0.42(0.14,0.69)$ \\
\hline $5-13$ years & $\mapsto-1$ & $0.53(0.37,0.69)$ \\
\hline $14-15$ years & $\longmapsto \quad \bullet$ & $1.59(1.03,2.16)$ \\
\hline Household incom & & \\
\hline Lowest $25 \%$ & $\longmapsto$ & $0.99(0.67,1.32)$ \\
\hline Middle $50 \%$ & $\mapsto-1$ & $0.48(0.29,0.67)$ \\
\hline Highest $25 \%$ & $\longmapsto$ & $0.40(0.16,0.64)$ \\
\hline School day* & & \\
\hline not schoolday & $\mapsto-1$ & $0.50(0.33,0.66)$ \\
\hline schoolday & $\longmapsto$ & $0.67(0.46,0.88)$ \\
\hline Overall & $H$ & $0.52(0.39,0.65)$ \\
\hline & $\begin{array}{llll}0.50 & 1.00 & 1.50 & 2.00 \\
\text { coefficient }(95 \% \mathrm{CI})\end{array}$ & 2.50 \\
\hline
\end{tabular}

Fig. 2 Association between meeting individual movement guidelines and HRQOL in subgroups. Note * 'Whether a school-day' data are available for 4-15 years old. All other data are available for 2-15 years old. In this figure, the model treated guideline adherence as a continuous variable: the total number of guidelines met. All the covariates in the primary analysis except the grouping variable were included in the subgroup analyses. The covariates include special health care needs, female, Indigenous status, speak English at home, two parent family, parental education, number of siblings, age group, and income group explanation for the stronger association with meeting physical activity guidelines would be its associated benefit in reducing screen time, building social connectedness, enhancing selfesteem and increasing sleep quality, which interact to result in better psychosocial health [7]. Teenagers aged $14-15$ years and children from low household income families showed stronger relationship between time use and HRQOL. The results provided additional insight on the possible HRQOL impact for children in environment that restricts physical activities such as the unexpected lockdown during the COVID-19 pandemic.

Associations between time use behavior and HRQOL appear to vary according to different development stages. For 2-4 years old, it appears that none of the three guidelines can be neglected, namely that only when all three guidelines were met can a HRQOL improvement be expected. In children aged 14-15 years, meeting all movement guidelines has the highest HRQOL benefit comparing to meeting none, with the HRQOL difference clinically meaningful (4.44 points differences which approaches the 4.50 points minimal clinically important difference) [17]. Zhu (2019) also found that failing to meet these guidelines had stronger associations with depression and anxiety in 12-17 years compared to 6-11 years [49].

Guidelines adherence was poorer in children from lowincome families, and the HRQOL improvement associated

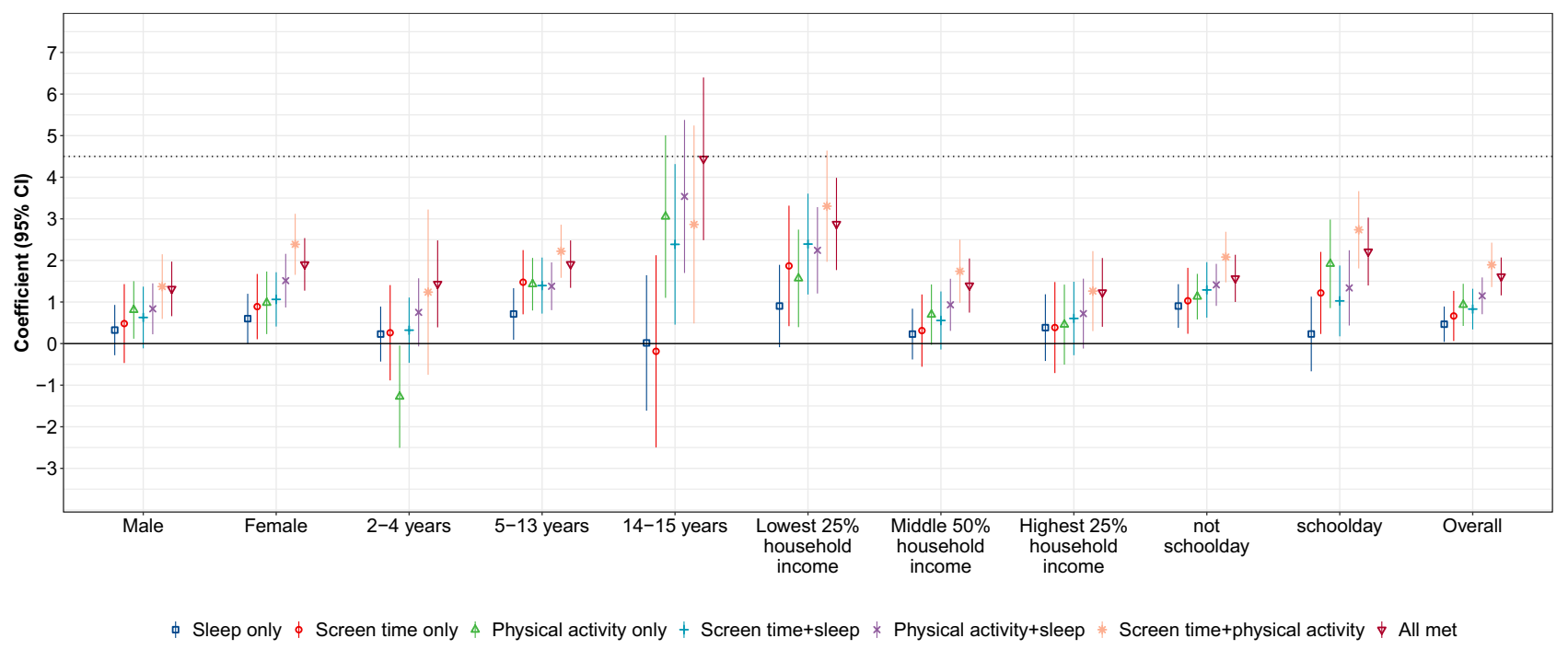

Fig. 3 Association between meeting combinations of movement guidelines and HRQOL in subgroups. Note In this figure, the model treated guideline adherence as a categorical variable: meeting different combinations of three guidelines: none, sleep, screen, physical activity, sleep and screen, sleep and physical activity, screen and physical activity, all three. Reference group is "none". "Whether a school-day' data are available for 4-15 years old. All the covariates in the primary analysis except the grouping variable were included in the subgroup analyses. The covariates include special health care needs, female, Indigenous status, speak English at home, two parent family, parental education, number of siblings, age group, and income group 
with guidelines adherence was higher for this group. Although guideline adherence is likely to contribute to the HRQOL, an caveat is that economically disadvantaged children could also have poorer physical health [40] which might also have a direct impact on HRQOL. The finding thus needs to be interpreted with caution where it is important to understand the interactions of socioeconomic status and health.

Strengths of our study include employing a large nationally representative dataset with a wide age range and measuring guideline adherence by time use diaries, which tends to provide 'the most accurate and comprehensive information' [50]. It has a potential advantage over simple recall questions in accuracy (intentionally over- or under-reporting or memory error in simple recall questions) [32, 33]. Its objectivity also enables comparability across countries and over time [51]. We have performed a rich set of subgroup analyses, which may provide more detailed information for targeted groups.

Several limitations are identified. While we had access to time use dairy data on physical activity, it is known that accelerometry data is more accurate, especially at capturing low intensity exercise [52], which could be important particularly for the 2-4 year age group. However, it is often not practical for population studies with large sample sizes and repeated survey design to adopt accelerometry measurement due to excessive cost $[52,53]$. Due to data availability and the aim of matching to 24-h guidelines, we tried to choose the best measure and take advantage of the unique time use diary data to answer the research question. We acknowledge that there are other tools to measure time use behavior and it would be interesting to compare the results when data are available. Most of the effect sizes in our study did not reach minimal clinically important difference. However smaller effect sizes may still be important at a population level or to groups with greater vulnerability [54]. The causal relationship between guideline adherence and HRQOL could not be determined in this study. Aiming to explore the agespecific effects of guideline adherence for a wider range of child ages, we did not explore whether previous adherence impacts future outcomes. Future research on this would be valuable. The screen time use guideline in our study focused on the recreational screen time, which is consistent with the current guideline. However, non-recreational screen time such as online learning, is increasingly used in education. Future studies on non-recreational screen use may be warranted especially considering the fast-changing movement behavior norms.

\section{Conclusions}

While meeting physical activity guidelines alone has the strongest association with HRQOL compared with meeting screen and sleep time guidelines alone, the integration of screen time and physical activity guidelines is associated with the highest HRQOL increment. The association between guidelines adherence and HRQOL appears strongest for adolescents, and those from low-income families.

Supplementary Information The online version contains supplementary material available at https://doi.org/10.1007/s11136-021-02901-6.

Author contributions XX conceptualized the study, performed data analyses, and drafted the manuscript; LH, KD, and NC conceptualized the study, provided input into the data analyses, and critically reviewed and revised the manuscript. RX provided input into the data analyses and reviewed and revised the manuscript. All authors read and approved the final manuscript.

Funding XX was supported by China Scholarship Council (201906010310). RX was supported by China Scholarship Council (201806010405). The sponsor was not involved in the study design, data collection, data analyses, results interpretation, and writing of this manuscript.

Data availability Researchers who are interested in using the data could apply at the LSAC website with study protocols (https://dataverse.ada. edu.au/dataverse/ada?q=LSAC).

\section{Declarations}

Conflict of interest We declare no actual or potential competing financial interests.

Ethical approval Appropriate approval was obtained from the Department of Social Services (Australia) to access the publicly available, de-identified longitudinal dataset.

\section{References}

1. Tremblay, M. S., et al. (2012). Canadian sedentary behaviour guidelines for the early years (aged 0-4 years). Applied Physiology, Nutrition and Metabolism, 37(2), 370-391.

2. Tremblay, M. S., et al. (2012). Canadian physical activity guidelines for the early years (aged 0-4 years). Applied Physiology, Nutrition, and Metabolism, 37(2), 345-356.

3. Health, U. D. O. (2011). Start active, stay active: a report on physical activity for health from the four home countries' chief medical officers. Crown Copyright London.

4. Australian Government Department of Health. (2010). Move and play every day national physical activity recommendations for children 0-5 years. Australian Government, Department of Health.

5. Marker, A. M., Steele, R. G., \& Noser, A. E. (2018). Physical activity and health-related quality of life in children and adolescents: A systematic review and meta-analysis. Health Psychology, 37(10), 893-903. 
6. Poitras, V. J., et al. (2016). Systematic review of the relationships between objectively measured physical activity and health indicators in school-aged children and youth. Applied Physiology, Nutrition, and Metabolism, 41(6), S197-S239.

7. Lubans, D., et al. (2016). Physical activity for cognitive and mental health in youth: A systematic review of mechanisms. Pediatrics, 138(3).

8. Lissak, G. (2018). Adverse physiological and psychological effects of screen time on children and adolescents: Literature review and case study. Environmental Research, 164, 149-157.

9. Stiglic, N., \& Viner, R. M. (2019). Effects of screentime on the health and well-being of children and adolescents: A systematic review of reviews. British Medical Journal Open, 9(1), e023191.

10. Chaput, J. P., et al. (2016). Systematic review of the relationships between sleep duration and health indicators in school-aged children and youth. Applied Physiology, Nutrition and Metabolism, 41(6 Suppl 3), S266-S282.

11. Chaput, J. P., et al. (2017). Systematic review of the relationships between sleep duration and health indicators in the early years (0-4 years). BMC Public Health, 17(Suppl 5), 855.

12. Tremblay, M. S., et al. (2016). Canadian 24-hour movement guidelines for children and youth: An integration of physical activity, sedentary behaviour, and sleep. Applied Physiology, Nutrition and Metabolism, 41(6 Suppl 3), S311-S327.

13. Tremblay, M. S. (2020). Introducing 24-hour movement guidelines for the early years: A new paradigm gaining momentum. Journal of Physical Activity \& Health, 17(1), 92-95.

14. Lang, C., et al. (2016). The relationship between physical activity and sleep from mid adolescence to early adulthood. A systematic review of methodological approaches and meta-analysis. Sleep Medicine Reviews, 28, 32-45.

15. Park, S. (2014). Associations of physical activity with sleep satisfaction, perceived stress, and problematic Internet use in Korean adolescents. BMC Public Health, 14(1), 1143.

16. Magee, C. A., Lee, J. K., \& Vella, S. A. (2014). Bidirectional relationships between sleep duration and screen time in early childhood. JAMA Pediatrics, 168(5), 465-470.

17. Varni, J. W., et al. (2003). The PedsQL $\mathrm{LM}^{\mathrm{T}} 4.0$ as a pediatric population health measure: Feasibility, reliability, and validity. Ambulatory Pediatrics, 3(6), 329-341.

18. Fayers, P. M., \& Machin, D. (2013). Quality of life: The assessment, analysis and interpretation of patient-reported outcomes. Wiley.

19. Guyatt, G. H., Feeny, D. H., \& Patrick, D. L. (1993). Measuring health-related quality of life. Annals of Internal Medicine, 118(8), 622-629.

20. Dolan, P. (2000). The measurement of health-related quality of life for use in resource allocation decisions in health care. Handbook of health economics, 1, 1723-1760.

21. Wilson, I. B., \& Cleary, P. D. (1995). Linking clinical variables with health-related quality of life. A conceptual model of patient outcomes. JAMA, 273(1), 59-65.

22. Didsbury, M. S., et al. (2016). Socio-economic status and quality of life in children with chronic disease: A systematic review. Journal of Paediatrics and Child Health, 52(12), 1062-1069.

23. Mohler-Kuo, M., \& Dey, M. (2012). A comparison of healthrelated quality of life between children with versus without special health care needs, and children requiring versus not requiring psychiatric services. Quality of Life Research, 21(9), 1577-1586.

24. Stalsberg, R., \& Pedersen, A. V. (2010). Effects of socioeconomic status on the physical activity in adolescents: A systematic review of the evidence. Scandinavian Journal of Medicine \& Science in Sports, 20(3), 368-383.
25. Welk, G. J., Wood, K., \& Morss, G. (2003). Parental influences on physical activity in children: An exploration of potential mechanisms. Pediatric exercise science, 15(1), 19-33.

26. Cleland, V., et al. (2011). A longitudinal study of the family physical activity environment and physical activity among youth. SAGE Publications.

27. Xiao, Q., et al. (2020). Sleep characteristics and health-related quality of life in 9- to 11-year-old children from 12 countries. Sleep Health, 6(1), 4-14.

28. Lacy, K. E., et al. (2012). Screen time and physical activity behaviours are associated with health-related quality of life in Australian adolescents. Quality of Life Research, 21(6), 1085-1099.

29. Motamed-Gorji, N., et al. (2019). Association of screen time and physical activity with health-related quality of life in Iranian children and adolescents. Health and Quality of Life Outcomes, 17(1), 2 .

30. Sampasa-Kanyinga, H., et al. (2017). Associations between meeting combinations of 24-h movement guidelines and health-related quality of life in children from 12 countries. Public Health, 153, $16-24$.

31. Hinkley, T., et al. (2020). Prospective associations with physiological, psychosocial and educational outcomes of meeting Australian 24-Hour Movement Guidelines for the Early Years. International Journal of Behavioral Nutrition and Physical Activity, 17(1), 36.

32. Boase, J., \& Ling, R. (2013). Measuring mobile phone use: Selfreport versus log data. Journal of Computer-Mediated Communication, 18(4), 508-519.

33. Fisher, R. J. (1993). Social desirability bias and the validity of indirect questioning. Journal of consumer research, 20(2), 303-315.

34. Soloff, C., Lawrence, D., \& Johnstone, R. (2005). LSAC sample design (Technical Paper No. 1). Australian Institute of Family Studies.

35. Baxter, J. (2007). Children's time use in the longitudinal study of Australian children: Data quality and analytical issues in the 4-year cohort. Australian Institute of Family Studies.

36. Tang, F. (2017). Random forest missing data approaches. University of Miami.

37. Corey, J., et al. (2014). The times of their lives: Collecting time use data from children in the longitudinal study of Australian children (LSAC). Retrived October, 2020, from https://growingupi naustralia.gov.au/sites/default/files/tp13.pdf

38. Liu, J., et al. (2015). Association among number, order and type of siblings and adolescent mental health at age 12. Pediatrics International, 57(5), 849-855.

39. Sanders, T., et al. (2019). Type of screen time moderates effects on outcomes in 4013 children: Evidence from the longitudinal study of Australian children. International Journal of Behavioral Nutrition and Physical Activity, 16(1), 117.

40. Spurrier, N. J., et al. (2003). Socio-economic differentials in the health-related quality of life of Australian children: Results of a national study. Australian and New Zealand Journal of Public Health, 27(1), 27-33.

41. Huang, L., Freed, G. L., \& Dalziel, K. (2020). Children with special health care needs: How special are their health care needs? Academic Pediatrics.

42. Fitzmaurice, G. M., \& Ravichandran, C. (2008). A primer in longitudinal data analysis. Circulation, 118(19), 2005-2010.

43. Humphrey, S. E., \& LeBreton, J. M. (2019). The handbook of multilevel theory, measurement, and analysis. American Psychological Association.

44. Bell, A., \& Jones, K. (2015). Explaining fixed effects: Random effects modeling of time-series cross-sectional and panel data. Political Science Research and Methods, 3(1), 133-153. 
45. Torres-Reyna, O. (2007). Panel data analysis fixed and random effects using Stata (v. 4.2). Data \& statistical services (Vol. 112). Priceton University.

46. Neuhaus, J. M., \& Kalbfleisch, J. D. (1998). Between-and withincluster covariate effects in the analysis of clustered data. Biometrics, 54(2), 638-645.

47. Danchin, M. (2021). Top 10 child health problems: What Australian parents think, RCH National Child Health Poll. The Royal Children's Hospital Melbourne. Retrived May 1, 2021, from https://www.rchpoll.org.au/polls/top-10-child-health-problemswhat-australian-parents-think/

48. Saunders, T. J., et al. (2016). Combinations of physical activity, sedentary behaviour and sleep: Relationships with health indicators in school-aged children and youth. Applied Physiology, Nutrition and Metabolism, 41(6 Suppl 3), S283-S293.

49. Zhu, X., Haegele, J. A., \& Healy, S. (2019). Movement and mental health: Behavioral correlates of anxiety and depression among children of 6-17 years old in the US. Mental Health and Physical Activity, 16, 60-65.

50. Harding, D.J. (1997). Measuring children's time use: A review of methodologies and findings. Center for Research on Child Wellbeing. Working paper, 97-1.
51. Bauman, A., Bittman, M., \& Gershuny, J. (2019). A short history of time use research; implications for public health. BMC Public Health, 19(2), 607.

52. Hinkley, T., et al. (2016). Preschool and childcare center characteristics associated with children's physical activity during care hours: An observational study. International Journal of Behavioral Nutrition and Physical Activity, 13(1), 117.

53. Pedišić, Ž, \& Bauman, A. (2015). Accelerometer-based measures in physical activity surveillance: Current practices and issues. British Journal of Sports Medicine, 49(4), 219.

54. Osoba, D., et al. (1998). Interpreting the significance of changes in health-related quality-of-life scores. Journal of clinical oncology, 16(1), 139-144.

Publisher's Note Springer Nature remains neutral with regard to jurisdictional claims in published maps and institutional affiliations. 